\title{
Response of Zeta Potential to the Fouling during Microfiltration of Surfactants
}

\author{
Kazuho Nakamura*, Satoshi Komaki, and Kanji Matsumoto \\ Yokohama National University Graduate School of Engineering, \\ Department of Chemical and Energy Engineering, \\ 79-5, Tokiwadai, Hodogaya-ku, Yokohama 240-8501, Japan
}

The response of zeta potential, $\zeta$ obs, to membrane surface condition and surfactants in solution was investigated from the viewpoint of the interaction between surfactant and membrane surface. The $\zeta_{\text {obs }}$ of three polyvinylidene difluoride (PVDF) microfiltration membranes, which had non-ionic hydrophilic, hydrophobic, and positively charged pore surfaces, with nominal pore size of $0.22 \mu \mathrm{m}$ were measured over wide range of the surfactants concentration. N-Dodecyltrimethyl ammonium chloride (DTAC) and Sodium Dodecyl Sulfate (SDS) were used as cationic and anionic surfactants, respectively. At the lower surfactant concentration (the conductivity below $20 \mathrm{mS} / \mathrm{m}$ ) the behaviors of $\zeta$ obs mainly depended on the combination of membrane and surfactant used and they can be explained by the interaction between surfactant and pore surface. At the higher surfactant concentration the behaviors of $\zeta$ obs mainly depended on type of surfactant rather than on membrane used. In the case of SDS the severe flux decline was observed at a certain concentration in all the membranes and the absolute value of $\zeta$ obs decreased and approached to zero reflecting the flux decline.

Key words : zeta potential / fouling $/$ microfiltration $/$ surfactants

\section{Introduction}

In the membrane filtration processes the fouling phenomena are considered as the major problems in many applications and are caused by various components existing in the feed stream. Countermeasures against the fouling have been studied in terms of the development of anti-fouling membrane materials ${ }^{1 \sim 4)}$, pretreatments of feed solution for removing or altering the foulant ${ }^{5 \sim 77}$, and the optimizing of designs of membrane module, processes, and operating and cleaning conditions $^{8 \sim 10)}$.

The fouling will be caused by physico-chemical interactions between the membrane and foulant and the main interactions are recognized as hydrophobic and electrostatic interactions. The water layer adjacent to the hydrophobic surface has a higher value of free

\footnotetext{
* Corresponding author

Tel: $+81-45-339-3980$

Fax: +81-45-339-4012

E-mail: naka1@ynu.ac.jp
}

energy than that of the bulk water and adsorption of foulants is favorable because it reduces the total free energy of the system. A water layer next to hydrophilic surface can have a lower value of free energy than the bulk water and it interferes the adsorption of foulants on the surface. The hydrophilic surface modification of membranes has been attempted for applying anti-fouling property to membrane ${ }^{1 \sim 4}$ ). Electrostatic interactions arise from the interaction between fixed charge and/or fixed dipoles of membrane surface and foulant in aqueous solution. The origin of these surface charges is considered as the adsorption of specific positive or negative ions and/or ionization of specific chemical group on their surface.

The charge property of porous membrane can be characterized by zeta potential and the trans-membrane streaming potential (SP) method. HelmholtzSmoluchowski (HS) equation has been usually used for determination of the zeta potential assuming that the pore has a cylindrical capillary with laminar flow and the surface conductivity and the electro double layer (EDL) overlapping in the pore are negligible ${ }^{11}$, 
Table 1 Features of surfactants

\begin{tabular}{lccc} 
& Chemical formula & $\begin{array}{c}\text { Molecular weight } \\
\left(\mathrm{g} \mathrm{mol}^{-1}\right)\end{array}$ & $\begin{array}{c}\text { Critical micelle } \\
\left.\text { concentration(CMC) }\left(25^{\circ} \mathrm{C}\right) 28\right)\end{array}$ \\
\hline DTAC & $\mathrm{C}_{12} \mathrm{H}_{25}-\mathrm{N}\left(\mathrm{CH}_{3}\right)_{3} \mathrm{Cl}$ & 263.9 & $\begin{array}{c}19.3 \mathrm{mM} \\
(5.09 \mathrm{~g} / \mathrm{L})\end{array}$ \\
\hline \multirow{2}{*}{ SDS } & $\mathrm{C}_{12} \mathrm{H}_{25}-\mathrm{OSO}_{3} \mathrm{Na}$ & 288.4 & $\begin{array}{c}8.13 \mathrm{mM} \\
(2.34 \mathrm{~g} / \mathrm{L})\end{array}$ \\
\hline
\end{tabular}

$$
\zeta_{\mathrm{obs}}=\frac{\mu \lambda}{\varepsilon_{0} \varepsilon_{\mathrm{r}}} \mathrm{SP}
$$

where $\varepsilon_{0}$ is the permittivity of free space, $\varepsilon_{\mathrm{r}}$ is relative dielectric constant, $\lambda$ is electric conductivity, and $\mu$ is viscosity. However, the thickness of EDL cannot be negligible compared with pore size range of MF membranes especially in lower ion concentration. HS equation can underestimate the zeta potential because of the overlapping in pore and the surface conductivity ${ }^{12}$. Thus, the zeta potential estimated from HS equation is "apparent" or "observed" one. However, it is useful for practical application because of its simple formulation.

Surfactant, which contains both hydrophilic groups and hydrophobic groups, plays important roles in membrane and membrane processes. In developing of membrane material surfactants are used as an additive to membrane polymer and surface modification agent for the formation of anti-fouling surface ${ }^{1 \sim 4}$. In membrane filtration process surfactant has been recognized as foulant of reverse osmosis (RO) or nanofiltration (NF) membranes ${ }^{12 \sim 15)}$, and it has been used as cleaning agents of fouled membranes, and separation agents in micellar-enhanced membrane processes ${ }^{16 \sim 19)}$ and oily waste water treatment process ${ }^{13,20 \sim 25)}$. The behaviors of surfactants during filtration are of interest for efficiencies of the membrane separation processes.

In this study the response of $\zeta$ obs to membrane surface condition and surfactants in solution were investigated from the viewpoint of the interaction between surfactant and membrane surface. The $\zeta$ obs of three polyvinylidene difluoride (PVDF) MF membranes, which had non-ionic hydrophilic, hydrophobic, and positively charged pore surfaces, with nominal pore size of $0.22 \mu \mathrm{m}$ were measured. N-Dodecyltrimethyl ammonium chloride (DTAC) and Sodium Dodecyl Sulfate (SDS) were used as cationic and anionic surfactants, respectively. A high throughput measurement of $\mathrm{SP}^{26)}$ was conducted in order to measure $\zeta$ obs over a

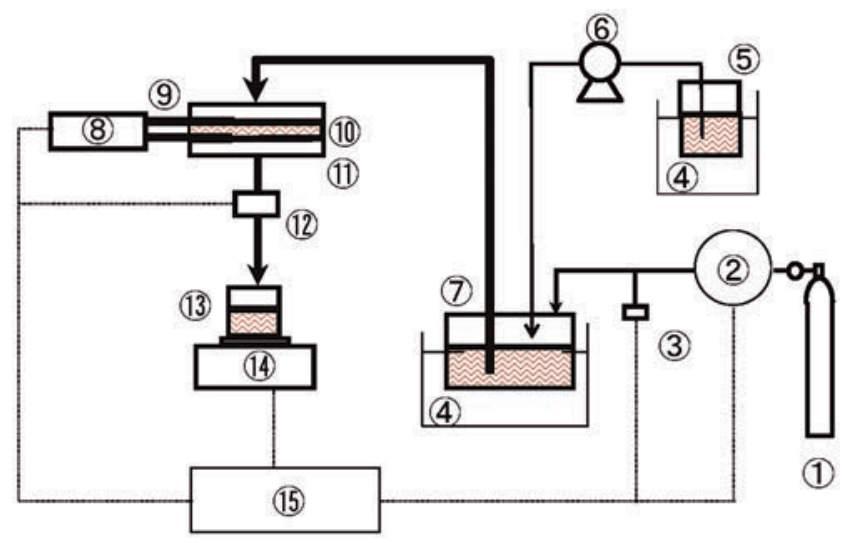

Fig. 1 Experimental apparatus ; (1) $\mathrm{N}_{2}$ cylinder, (2) pressure regulator, (3) pressure transducer, (4) thermo bath, (5) feed tank, (6) pump, (7) feed mixing tank, (8) digital multi-meter, (9) Pt-black electrode, (10) membrane, (11) membrane holder, (12) conductivity cell, (13) beaker, (14) electric balance, (15) personal computer.

wide range of surfactant. At first $\zeta$ obs of three PVDF membranes was studied using $\mathrm{KCl}$ solution and then the effects of the surfactants on the zeta potential were discussed.

\section{Experimental}

\subsection{Membranes and chemicals}

PVDF microfilration (MF) membranes having three different surface chemistry, which were hydrophilic (GVWP Durapore, Millipore), hydrophobic (GVHP Durepore, Millipore), and positively charged hydrophilic (Charged Durepore, Millipore), with a nominal pore size of $0.22 \mu \mathrm{m}$ were used. The only difference among these membranes was the surface chemistry. According to manufacturer's patent ${ }^{27)}$ and catalog the hydrophilic membrane has no ionized group on the pore surface and the hydrophobic membrane is made of plain PVDF and the positively charged membrane has positive ionizable group on the pore surface.

Potassium chloride $(\mathrm{KCl})$ (Wako Pure Chemical Industries) solution was used as a non-surface active 

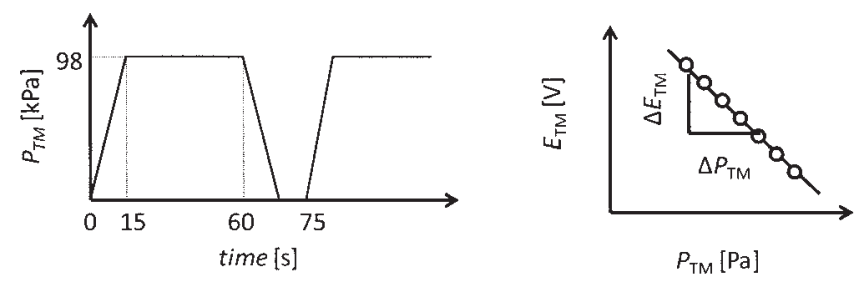

Fig. 2 The pulse pattern of applied pressure and the definition of streaming potential.

electrolyte solution. $\mathrm{KCl}$ solution was prepared with ultra pure water and filtered with a $0.5 \mu \mathrm{m}$ membrane filter before use. N-Dodecyltrimethyl ammonium chloride (DTAC) (Wako Pure Chemical Industries) and Sodium Dodecyl Sulfate (SDS) (Wako Pure Chemical Industries) were used as cationic and anionic surfactants, respectively. The features of these surfactants were shown in Table 1.

\subsection{Experimental apparatus}

Fig. 1 shows the experimental apparatus. A deadend membrane housing (25 $\mathrm{mm}$ diameter, effective filtration area $3.0 \mathrm{~cm}^{2}$ ) with platinum black electrodes for streaming potential measurement was used. The permeate flux, $J$, filtration pressure, $P_{\mathrm{TM}}$, electrical potential across membrane, $E_{\mathrm{TM}}$, electric conductivity, $\lambda$, of filtrate were measured with a electric balance (Shimadzu, EB3300SW), pressure transducer (Kyowa, PVL-5KB), digital multi-meter (Advantest, R6441), electro conductivity meter (TOADKK, CM40G), respectively. The measured data were recorded by a personal computer. The temperature in the feed tank and the mixing tank was kept at 298K. During filtration the concentration of the feed component in the feed mixing tank can be increased continuously by adding feed component to the mixing tank.

Fig. 2 shows the pulse pattern of applied pressure for SP measurement during filtration and the definition of SP. All filtration experiments were conducted with this pulse pattern pressure.

The SP was defined as the slope of linear regression for $E_{\mathrm{TM}}$ versus $P_{\mathrm{TM}}$ plots, as $\Delta E_{\mathrm{TM}} / \Delta P_{\mathrm{TM}}$.

$$
\text { Streaming Potential (SP) }=\frac{\Delta E_{\mathrm{TM}}}{\Delta P_{\mathrm{TM}}}
$$

The $E_{\text {TM }}$ versus $P_{\text {TM }}$ plots showed good linearity, showing the validity of this measurement.

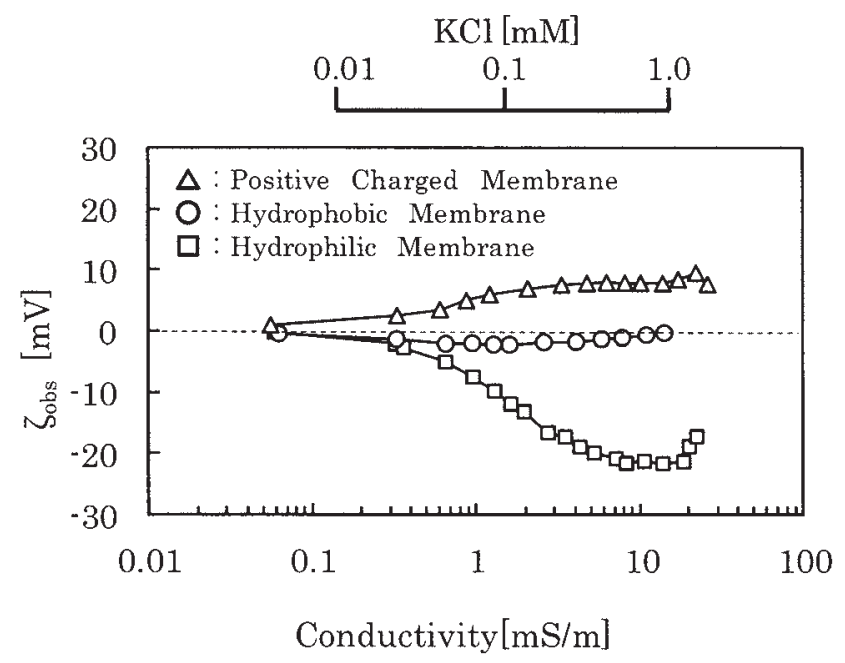

Fig. 3 Effects of $\mathrm{KCl}$ concentration on $\zeta$ obs of hydrophilic, hydrophobic, and positively charged membranes.

\subsection{Filtration procedure}

Membrane was wetted by alcohol before use and set to the membrane holder. Flux and SP were measured with pure water. And then the filtration was conducted by the pulse pattern pressure. During the filtration the concentration of the feed component in the feed mixing tank was gradually increased by adding the feed component. SP and flux were measured during the filtration.

\section{Results and discussion}

The $\zeta_{\text {obs }}$ will depend on the surface charge, pore size, and conductivity in pore. At first $\zeta$ obs was measured with $\mathrm{KCl}$ solution and the effect of the conductivity on $\zeta$ obs was discussed. And then $\zeta$ obs was measured with SDS and DTAC solutions and $\zeta$ obs was discussed from the view point of the charge condition of pore surface. During the measurements the rejection of surfactants, which was determined by conductivity, was not observed. The adsorption of surfactant on membrane can be characterized by $\zeta_{\text {obs }}{ }^{29 \sim 31)}$. It is considered that the change in $\zeta_{\text {obs }}$ is due to the adsorption of surfactants in this study, although there was no direct evidence of the adsorption because the change in concentration of surfactants by the adsorption was too small to detect during the adsorption process.

\subsection{Effect of $\mathrm{KCl}$ concentration on $\zeta$ obs}

Fig. 3 shows the effect of $\mathrm{KCl}$ concentration on $\zeta$ obs 

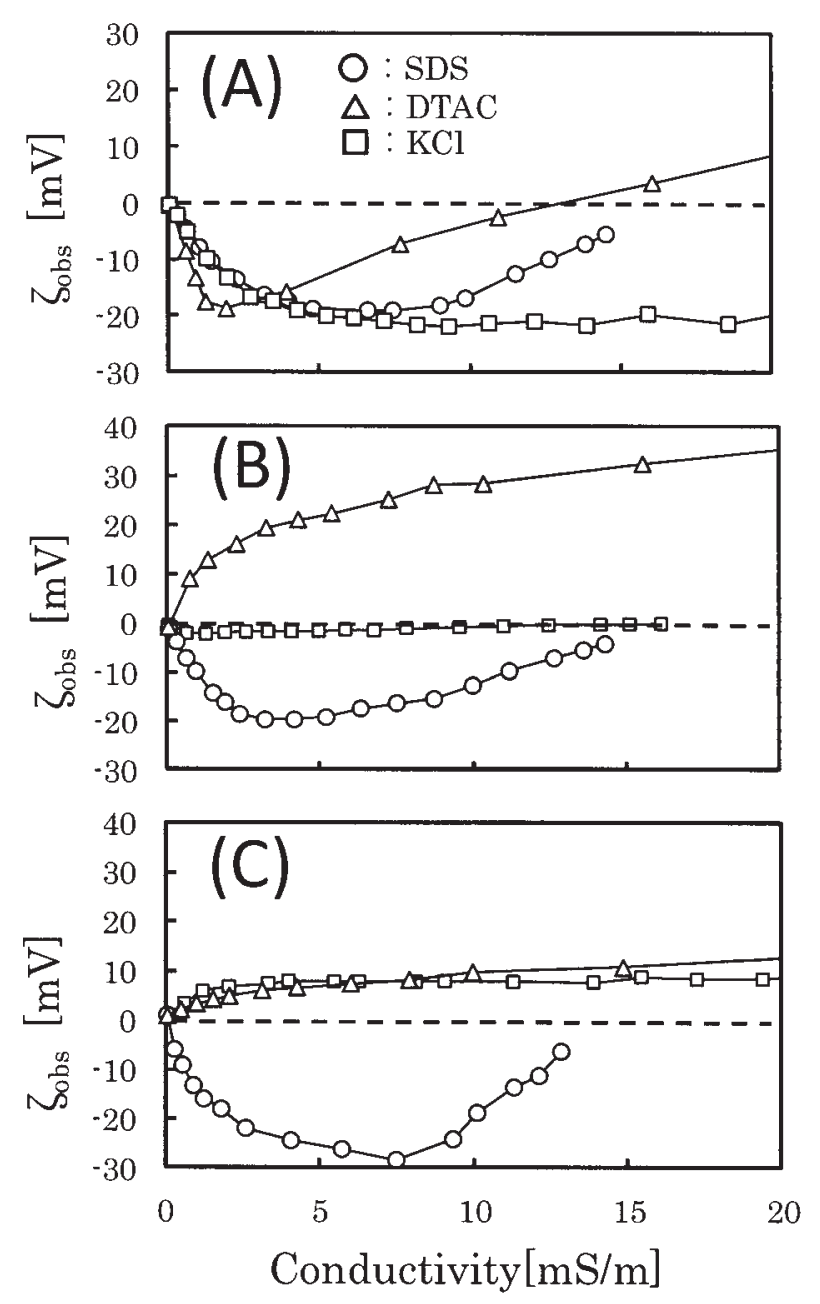

Fig. 4 Effects of concentration of surfactants on $\zeta$ obs in the three membranes. (A) hydrophilic membrane, (B) hydrophobic membrane, (C) positively charged membrane with SDS, DTAC and $\mathrm{KCl}$ solutions.

in the three PVDF membranes. In the hydrophilic membrane the curve of $\zeta$ obs showed negative charge and the concave down shape. The absolute value of $\zeta$ obs at the conductivity below $5 \mathrm{mS} / \mathrm{m}$ would be lowered due to the overlapping of EDL in the pore. As this hydrophilic pore surface has no ionizable groups the origin of the negative charge would be adsorption of anion, i.e. chloride ion, on the pore surface. The hydrophobic membrane, of which surface chemical composition is plain PVDF carrying no ionizable groups, showed very little negative charge. These results show that the hydrophobic surface has little affinity to chloride ion compared with the hydrophilic surface. The positively charged membrane showed positive charge deriving from the surface ionizable group. It was seemed that in the hydrophobic and the positively charged membranes the adsorption of ions

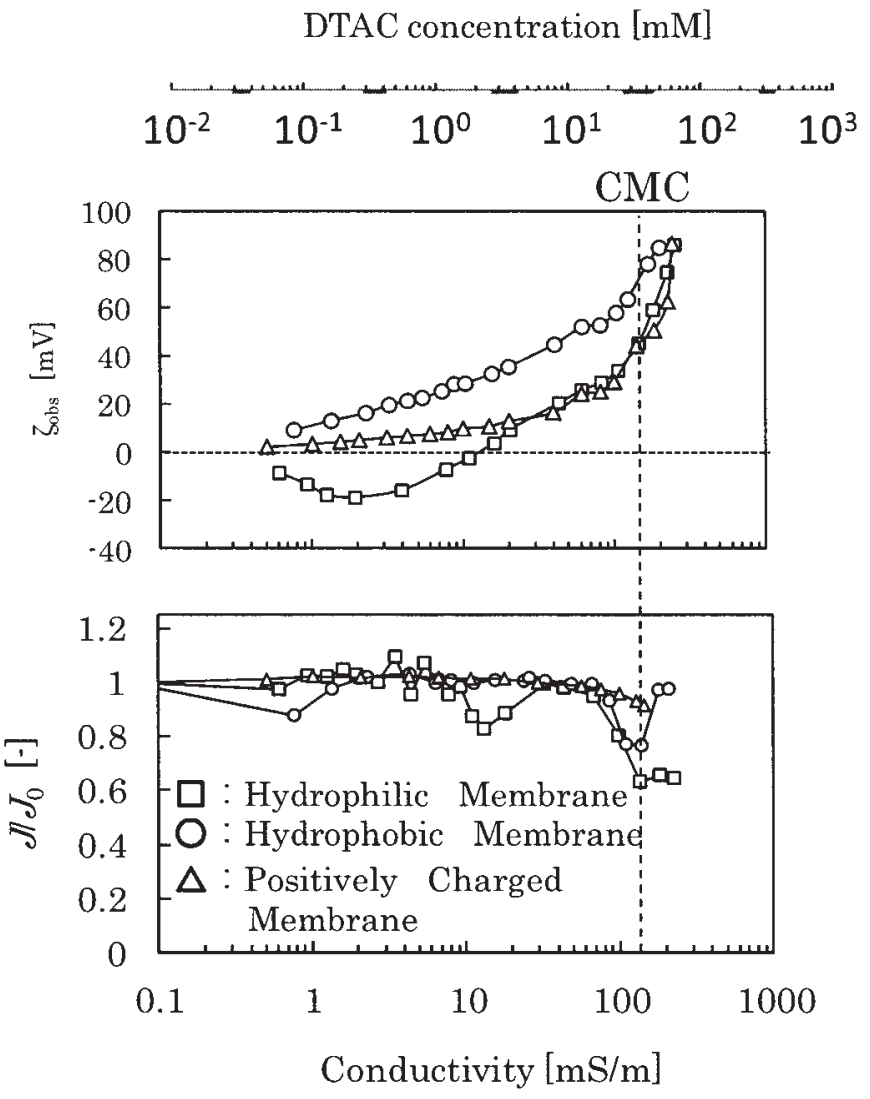

Fig. 5 Effect of DTAC concentration on $\zeta$ obs and flux of the hydrophilic, hydrophobic and positively charged PVDF membranes.

had little impact on the origin of the charge.

It is expected that the absolute value of $\zeta$ obs will decrease in the higher ionic strength due to compression of EDL ${ }^{11}$. The absolute value of $\zeta$ obs of the positively charged and hydrophilic membranes decreased in the conductively above $20 \mathrm{mS} / \mathrm{m}$ although the experimental data in much higher conductivity could not be measured because the $E$ TM was too small to measure by the experimental app aratus.

\subsection{Effect of surfactant on $\zeta_{\text {obs }}$ in the lower surfactant concentration (conductivity below $20 \mathrm{mS} / \mathrm{m}$ )}

It is expected that the surface charge density of pore surface will be drastically altered by the adsorption of surfactants. The charge condition of pore surface will be altered to positive by dodecyltrimethyl ammonium (DTA) ion and to negative by dodecyl sulfate (DS) ion. The $\zeta_{\text {obs }}$ of the three PVDF membranes were measured using DTAC solution and SDS solution. In this section the effect of surfactants on $\zeta$ obs at the conductivity below $20 \mathrm{mS} / \mathrm{m}$ was discussed. Fig. 4 shows the 


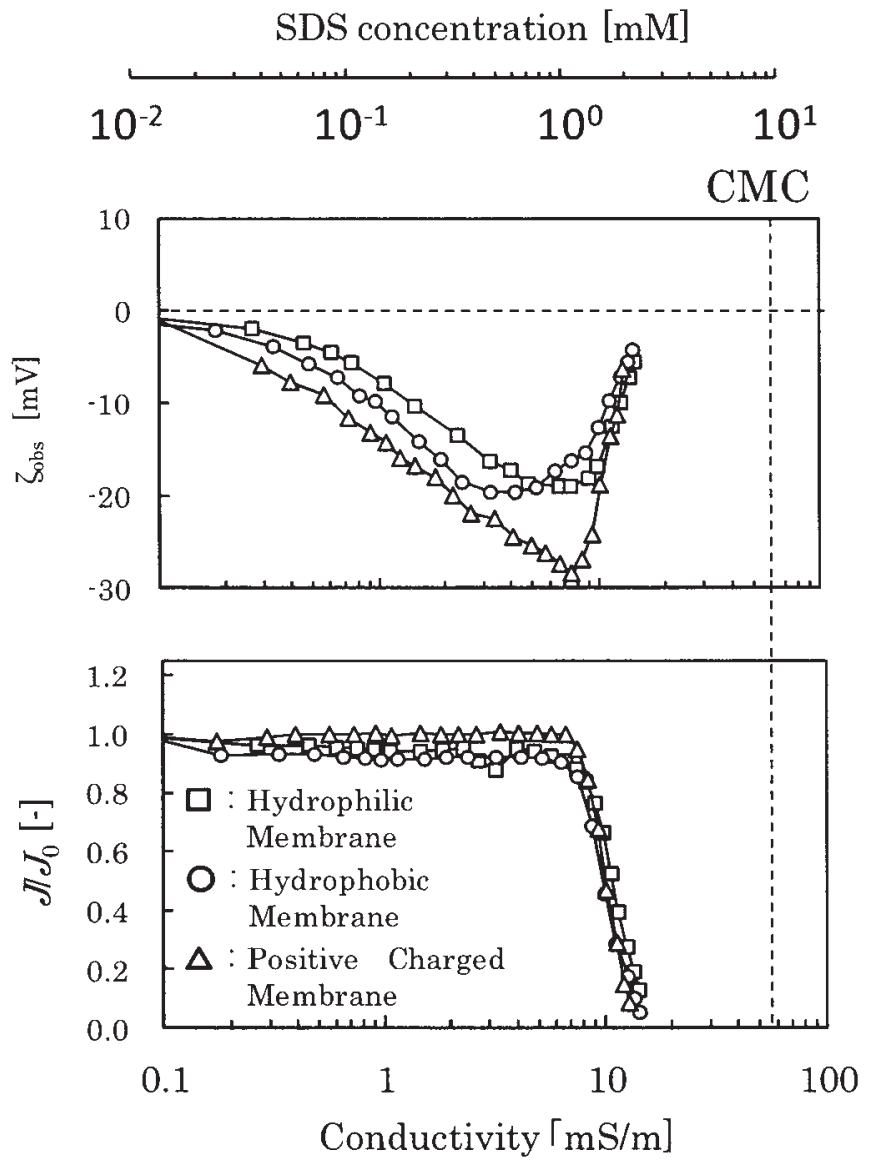

Fig. 6 Effect of SDS concentration on $\zeta_{\text {obs }}$ and flux of the hydrophilic, hydrophobic and positively charged PVDF membranes.

effects of the SDS, DTAC and $\mathrm{KCl}$ concentration on $\zeta_{\text {obs }}$ in the three membranes. Although in this concentration range the absolute value of $\zeta$ obs should be affected by the overlapping of EDL, the sign of $\zeta$ obs will reflect the sign of surface charge.

In the hydrophilic membrane ((A) in Fig. 4) $\zeta$ obs showed negative value in all the solution at the conductivity below $10 \mathrm{mS} / \mathrm{m}$. The origin of these negative charges would be adsorption of anions, which are chloride ion in DTAC and $\mathrm{KCl}$ solutions and dodecyl sulfate (DS) ion in SDS solution. In the case of DTAC solution $\zeta$ obs showed a minimum at about $2 \mathrm{mS} / \mathrm{m}$ and became positive at the conductivity above $15 \mathrm{mS} / \mathrm{m}$. This increase in $\zeta$ obs would reflect the increase in DTA adsorption. The condition of the minimum was not clear from these observations although it is expected that the minimum would reflect the competitive adsorption of anions and DTA. In the case of SDS solution $\zeta$ obs showed almost same behavior as that in $\mathrm{KCl}$ solution in the conductivity below $5 \mathrm{mS} / \mathrm{m}$ and increased after showing a minimum at around $6 \mathrm{mS} / \mathrm{m}$.
This increase will be discussed later combined with flux decline.

In the hydrophobic membrane ((B) in Fig. 4) $\zeta_{\text {obs }}$ clearly showed the same sign of ions of surfactants in this concentration range, which are positive in DTAC solution and negative in SDS solution. These results suggest that the surfactants will adsorb on the hydrophobic surface by hydrophobic interaction and provide the charges. In the case of SDS solution $\zeta_{\text {obs }}$ showed a minimum and this behavior will be discussed later.

In the positively charged membrane ((C) in Fig. 4) $\zeta$ obs showed positive value in the cases of DTAC and $\mathrm{KCl}$ solutions and negative value in the case of SDS solutions in this concentration range. In the cases of DTAC and $\mathrm{KCl}$ solutions the origin of the positive charges would be the surface ionizable group or adsorption of DTA ion. In the case of SDS solution the origin of the negative charge would be DS ion. This result suggests that the DS will adsorb on the positive charged surface by electrostatic attractive interaction and provide negative charge on the surface. In the case of SDS solution $\zeta$ obs showed a minimum and this behavior will be discussed later.

From these observations in the concentration range below $20 \mathrm{mS} / \mathrm{m}$ the behaviors of $\zeta$ obs depended on the combination of surfactant and membrane.

\subsection{Effect of surfactant on $\zeta_{\text {obs }}$ in the higher surfactant concentration}

Fig. 5 shows the effects of the DTAC concentration on $\zeta$ obs and flux in the three membranes in the conductivity range from $0.5 \mathrm{mS} / \mathrm{m}$ to $200 \mathrm{mS} / \mathrm{m}$. The severe flux decline was not observed although the flux varied at around the CMC of DTAC. $\zeta$ obs increased with the increase in DTAC concentration at conductivity above $10 \mathrm{mS} / \mathrm{m}$. This result suggests that the amount of adsorbed DTA ion would increase with the increase in DTAC concentration regardless of membrane used. The $\zeta_{\text {obs }}$ of the hydrophobic membrane was higher than those of the hydrophilic and positively charged membranes over the range of concentration studied. This result means that the hydrophobic interaction would enhance the amount of adsorbed surfactant. The $\zeta_{\text {obs }}$ at around the conductivity of $200 \mathrm{mS} / \mathrm{m}$ showed almost same value in the all three membranes.

Fig. 6 shows the effects of surfactant concentration on $\zeta$ obs and flux in the filtration of SDS solution with 
the three membranes in the conductivity range from $0.5 \mathrm{mS} / \mathrm{m}$ to $15 \mathrm{mS} / \mathrm{m}$. In the conductivity below 7 $\mathrm{mS} / \mathrm{m}$ the flux decline was not observed. At the conductivity of about $7 \mathrm{mS} / \mathrm{m}$ the flux suddenly decreased in all the membranes. The reason of this flux decline, i.e. fouling, is not clear. It was not possible to measure $\zeta$ obs in much higher SDS concentration because of this flux decline.

The $\zeta$ obs showed negative over the concentration range in all the membranes, suggesting the adsorption of chloride ion and DS ion on the pore surface. In the conductance below $7 \mathrm{mS} / \mathrm{m}$ the thickness of the adsorption layer would be much smaller than pore size of these membranes because the flux decline was not observed in this concentration range. In all the membranes the $\zeta$ obs decreased in the lower conductivity and increased and approached to zero after showing a minimum. The SDS concentration at the minimal zeta potential was almost same as the start point of the severe flux decline. The decrease in the absolute value of $\zeta$ obs due to fouling was observed in other filtration systems ${ }^{32}{ }^{35}$ ) and this phenomenon has not been fully interpreted. A possible examination is that the location reflecting $\zeta$ obs would shift from the membrane to the fouling part because the location of dominant pressure drop would shift from membrane to the fouling part and the $\zeta$ obs of the fouling part would be small value.

From these observations in the higher surfactant concentration the behavior of $\zeta$ obs depended mainly on type of surfactant rather than the type of membrane used.

\section{Conclusion}

The $\zeta$ obs of the three PVDF membranes, which have non-ionic hydrophilic, hydrophobic and positively charged pore surfaces, with nominal pore size 0.22 $\mu \mathrm{m}$ was observed using $\mathrm{KCl}$, DTAC and SDS solutions over wide range of the surfactants concentration. At the lower surfactant concentration (the conductivity below $20 \mathrm{mS} / \mathrm{m}$ ) the behaviors of $\zeta$ obs depended on the combination of membrane and surfactant used. At the higher surfactant concentration the behaviors of $\zeta$ obs mainly depended on type of surfactant rather than on membrane used. In the case of SDS the severe flux decline was observed at a certain concentration in all the membranes and the absolute value of $\zeta$ obs decreased and approached to zero reflecting the flux decline.

\section{Nomenclature Symbols}

$\begin{array}{llr}E_{\mathrm{TM}} & \text { electrical potential across } & \\ & \text { membrane } & (\mathrm{V}) \\ J & \text { permeate flux } & (\mathrm{m} / \mathrm{s}) \\ J_{0} & \text { permeate flux of pure water } & (\mathrm{m} / \mathrm{s}) \\ P_{\mathrm{TM}} & \text { filtration pressure } & (\mathrm{Pa}) \\ \varepsilon_{0} & \text { permittivity of free space } & (\mathrm{F} / \mathrm{m}) \\ \varepsilon_{\mathrm{r}} & \text { relative dielectric constant } & (-) \\ \lambda & \text { electric conductivity } & (\mathrm{S} / \mathrm{m}) \\ \mu & \text { viscosity } & (\mathrm{Pa} \mathrm{s}) \\ \zeta \text { obs } & \text { zeta potential obtained from } & \\ & \text { streaming potential measurement } & (\mathrm{V})\end{array}$

\section{References}

1) Yong-Hong Zhao, Daniel F. Shantz : Modified Anopore hybrid membranes for the microfiltration of cellulose acid hydrolysis mixtures, J. Membr. Sci., 377, 99-109 (2011)

2) Y. Mansourpanah, K. Alizadeh, S.S. Madaeni, A. Rahimpour, H. Soltani Afarani : Using different surfactants for changing the properties of poly(piperazineamide) TFC nanofiltration membranes, Desalination, 271, 169-177 (2011)

3) Elizabeth M. Van Wagner, Alyson C. Sagle, Mukul M. Sharma, Young-Hye La, Benny D. Freeman : Surface modification of commercial polyamide desalination membranes using poly(ethylene glycol) diglycidyl ether to enhance membrane fouling resistance, J. of Membr. Sci., 367, 273-287 (2011)

4) Nasrul Arahman, Tatsuo Maruyama, Tomohiro Sotani and Hideto Matsuyama : Fouling reduction of a poly (ether sulfone) hollow-fiber membrane with a hydrophilic surfactant prepared via non-solvent-induced phase separation, J. Appl. Polym. Sci., 111, 1653-1658 (2009)

5) Zsuzsanna László, Szabolcs Kertész, Sándor Beszédes, Zsuzsanna Hovorka-Horváth, Gábor Szabó, Cecilia Hodúr: Effect of preozonation on the filterability of model dairy wastewater in nanofiltration, Desalination, 240, 170-177 (2009)

6) Doherty, W. O. S.; Rackemann, D. W.; Steindl, R. J. : Fouling of tubular ceramic membranes during processing of cane sugar juice, Desalination and Water Treatment, 16, 45-56 (2010)

7) Chien-Hwa Yu, Lung-Chen Fang, Shaik Khaja Lateef, Chung-Hsin $\mathrm{Wu}$, Cheng-Fang Lin : Enzymatic treatment for controlling irreversible membrane fouling in crossflow humic acid-fed ultrafiltration, J. Hazard. Mater., 177, 1153-1158 (2010)

8) W.J.C. van de Ven, I.G.M. Pünt, A. Zwijnenburg, A.J.B. 
Kemperman, W.G.J. van der Meer, M. Wessling : Hollow fiber ultrafiltration: The concept of partial backwashing, J. Membr. Sci., 320, 319-324 (2008)

9) S. S. Madaeni, H. R. Tavakolian, F. Rahimpour : Cleaning Optimization of Microfiltration Membrane Employed for Milk Sterilization, Sep. Sci. Technol., 46, 571-580 (2011)

10) V. Chen, H. Li, D. Li, S. Tan, H.B. Petrus : Cleaning strategies for membrane fouled with protein mixtures, Desalination, 200, 198-200 (2006)

11) Leos J.Zeman, Andrew L. Zydney : Microfiltration and Ultrafiltration Principles and Applications, Mercel Dekker Inc., New York (1996)

12) Anthony Szymczyk, P. Fievet, B. Aoubiza, C. Simon, J. Pagetti : An application of the space charge model to the electrolyte conductivity inside a charged microporous membrane, J. Membr. Sci., 161, 275-285 (1999)

13) T. Srisukphun, C. Chiemchaisri, T. Urase, K. Yamamoto : Foulant interaction and RO productivity in textile wastewater reclamation plant, Desalination, 250, 845-849 (2010)

14) Katleen Boussu, Céline Kindts, Carlo Vandecasteele and Bart Van der Bruggen : Surfactant fouling of nanofiltration membranes: measurements and mechanisms, Chemphyschem., 8, 1836-1845 (2007)

15) Yasemin Kaya, Coskun Aydiner, Hulusi Barlas, Bulent Keskinler : Nanofiltration of single and mixture solutions containing anionics and nonionic surfactants below their critical micelle concentrations (CMCs), J. Membr. Sci., 282, 401-412 (2006)

16) Ü. Daniş, B. Keskinler : Chromate removal from wastewater using micellar enhanced crossflow filtration: Effect of transmembrane pressure and crossflow velocity, Desalination, 249, 1356-1364 (2009)

17) Ummuhan Danis, Coskun Aydiner : Investigation of process performance and fouling mechanisms in micellar-enhanced ultrafiltration of nickel-contaminated waters, J. Hazard. Mater., 162, 577-587 (2009)

18) Fu Zhao, Andres Clarens, and Steven J. Skerlos : Optimization of Metalworking Fluid Microemulsion Surfactant Concentrations for Microfiltration Recycling, Environ. Sci. Technol., 41, 1016-1023 (2007)

19) M. Muthukrishnan, B.K. Guha : Heavy metal separation by using surface modified nanofiltration membrane, Desalination, 200, 351-353 (2006)

20) Abdolhamid Salahi, Ali Gheshlaghi, Toraj Mohammadi, Sayed Siavash Madaeni : Experimental performance evaluation of polymeric membranes for treatment of an industrial oily wastewater, Desalination, 262, 235-242 (2010)

21) B. Hu, K. Scott : Microfiltration of water in oil emulsions and evaluation of fouling mechanism, Chem. Eng. J., 136, 210-220 (2008)

22) B. Hu, K. Scott : Influence of membrane material and cor- rugation and process conditions on emulsion microfiltration, J. Membr. Sci., 294, 30-39 (2007)

23) K. Boussu, C. Kindts, C. Vandecasteele, B. Van der Bruggen : Applicability of nanofiltration in the carwash industry, Sep. Purif. Technol., 54, 139-146 (2007)

24) Zhang Jin, Sun Yu-xin, Huang Zhi-feng, Liu Xing-qin, Meng Guang-yao : Treatment of phosphate-containing oily wastewater by coagulation and microfiltration, $J$. Envir. Sci., 1\&, 629-633 (2006)

25) T.Y. Chiu, A.E. James : Microfiltration of amphoteric surfactant using ceramic membranes, Colloid Surf. A Physicochem. Eng. Asp., 280, 58-65 (2006)

26) Nakamura K, Hui-yuan Liu, Matsumoto K : Effects of Pore size and Conductivity of Electrolyte Solution on the Measurement of Streaming Potential and Zeta potential of Microporous Membrane, Membrane (Maku), 29, 180187 (2004)

27) United States Patent, 5, 503, 746

28) Kaimenkasseizai (Surfactant) Handbook, Yoshida Tokiyuki (Eds.), Kougakutosyo, Tokyo (1987)

29) Sunjin Kim, Noeon Park, Sungyun Lee, Jaeweon Cho : Membrane characterizations for mitigation of organic fouling during desalination and wastewater reclamation, Desalination, 238, 70-77 (2009)

30) Kenichi Ikeda, Hisao Hachisuka, Toshitaka Nakamura, Shoji Kimura : Zeta-potential of fouled thin film composite membrane, J. Chem. Eng. Jpn, 32, 581-587 (1999)

31) Michelle Chapman Wilbert, John Pellegrino, Andrew Zydney : Bench-scale testing of surfactant-modified reverse osmosis/nanofiltration membranes, Desalination, 115, 15-32 (1998)

32) Myung-Suk Chun, Hong Il Cho, In Kyu Song : Electrokinetic behavior of membrane zeta potential during the filtration of colloidal suspensions, Desalination, 148, 363368 (2002)

33) Myung-Suk Chun, Wan Cheol Park : Time evolution of electrokinetic flow-induced streaming potential and flux in dead-end and cross flow filtration of colloids through nanopores, J. Membr. Sci., 243, 417-424 (2004)

34) Nadine Le Bolay, Alain Ricard : Streaming potential in membrane processes: Microfiltration of Egg proteins, $J$. Collid Interface Sci., 170, 154-160 (1995)

35) Laurence Ricq, Andŕe Pierre, Jean-Claude Reggiani, Serge Zaragoza-Piqueras, Jacques Pagetti, Georges Daufin : Effects of proteins on electrokinetic properties of inorganic membranes during ultra- and micro-filtration, J. Membr. Sci., 114, 27-38 (1996)

(Received 29 January 2012; Accepted 10 April 2012) 